ARTICLE

DOI: $10.1038 / \mathrm{s} 41467-018-02930-9$

\title{
A general soft-enveloping strategy in the templating synthesis of mesoporous metal nanostructures
}

Jixiang Fang ${ }^{1}$, Lingling Zhang ${ }^{1}$, Jiang $\mathrm{Li}^{1}$, Lu $\mathrm{Lu}^{2}$, Chuansheng $\mathrm{Ma}^{2}$, Shaodong Cheng ${ }^{2}$, Zhiyuan $\mathrm{Li}^{3}$, Qihua Xiong ${ }^{4} \&$ Hongjun You ${ }^{1}$

Metal species have a relatively high mobility inside mesoporous silica; thus, it is difficult to introduce the metal precursors into silica mesopores and suppress the migration of metal species during a reduction process. Therefore, until now, the controlled growth of metal nanocrystals in a confined space, i.e., mesoporous channels, has been very challenging. Here, by using a soft-enveloping reaction at the interfaces of the solid, liquid, and solution phases, we successfully control the growth of metallic nanocrystals inside a mesoporous silica template. Diverse monodispersed nanostructures with well-defined sizes and shapes, including Ag nanowires, 3D mesoporous Au, AuAg alloys, Pt networks, and Au nanoparticle superlattices are successfully obtained. The 3D mesoporous AuAg networks exhibit enhanced catalytic activities in an electrochemical methanol oxidation reaction. The current soft-enveloping synthetic strategy offers a robust approach to synthesize diverse mesoporous metal nanostructures that can be utilized in catalysis, optics, and biomedicine applications.

\footnotetext{
${ }^{1}$ Key Laboratory of Physical Electronics and Devices of the Ministry of Education, School of Electronic and Information Engineering, Xi'an Jiaotong University, Xi'an, Shannxi 710049, China. ${ }^{2}$ School of Microelectronics, Xi'an Jiaotong University, Xi'an, Shannxi 710049, China. ${ }^{3}$ College of Physics and Optoelectronic Engineering, South China University of Technology, Guangzhou 510640, China. ${ }^{4}$ Division of Physics and Applied Physics, School of Physical and Mathematical Sciences, Nanyang Technological University, Singapore, 637371, Singapore. Correspondence and requests for materials should be addressed to J.F. (email: jxfang@mail.xjtu.edu.cn) or to H.Y. (email: hjyou@mail.xjtu.edu.cn)
} 
S ince the discovery of ordered mesoporous silicas in the $1990 s^{1-3}$, ordered mesoporous materials have attracted much interest owing to their wide range of applications in biosensors, surface-enhanced spectroscopy, separation, drug delivery, catalysis, and fuel cells, which cannot be achieved by other compositions or structures ${ }^{4-8}$. These fascinating applications are inherent to metal frameworks with high porosities, large areas per unit volume, tunable pore sizes, narrow pore size distributions, high electroconductivities, and excellent activity-structure relationships. To date, mesoporous metals in bulk, thin film and powder forms have been exploited by means of electrochemical dealloying, soft-templating or hard-templating, and solution phase approaches ${ }^{9-13}$. Generally, soft-templating and hard-templating methods have achieved good pore size and structural control. However, the obtained morphologies of mesoporous metals have been limited to mainly powders with irregular morphology or films on conductive substrates ${ }^{14-16}$. These limitations are very serious for the further development of these metals since in some areas such as optical spectroscopy, catalysis, and biomedicine, the unambiguous identification of nanoparticle (NP) properties requires monodisperse NPs with a well-defined particle size, shape, composition, and crystal structure to avoid averaging effects ${ }^{17,18}$. In addition, the reliable performance in some applications requires consistent properties that are only possible with monodisperse NPs ${ }^{19,20}$.

To date, although a variety of routes have been developed to synthesize ordered mesoporous metals and metal oxides using mesoporous silica or carbon as a hard template ${ }^{21}$, in comparison with metal oxides, the synthesis of monodispersed ordered mesoporous metals (particularly in gold and silver) with wellcontrolled sizes and shapes has not yet been conducted by such approaches ${ }^{22,23}$. Only the preparation of mesoporous metals such as platinum, osmium and palladium has been reported ${ }^{15,24,25}$. In almost all the cases that utilize hard-templates, nanostructured $\mathrm{Au}$ materials have only been used as spherical NPs, nanosheets and nanowires, without $3 \mathrm{D}$ mesoporous structures ${ }^{26-28}$. This is partially because $\mathrm{Au}$ and $\mathrm{Ag}$ have relatively faster deposition rates than does $\mathrm{Pt}$ within the limited space of the mesoporous templates; these elements tend to grow rapidly along the dominated channels, and their growth in the micropores is insufficient ${ }^{29}$. In addition, negatively charged metallic species (ions, atoms, and cluster) have high mobilities inside mesoporous silica and thus prefer to migrate to the outside of the mesoporous silica during a reduction process, resulting in the formation of bulk particles and mixed morphologies (Fig. 1b) ${ }^{22}$. Therefore, in recent years, various protocols such as the surface functionalization of host silica $^{30}$, ion-exchange ${ }^{31}, \mathrm{CTA}-\mathrm{AuBr}_{4}$ complex formationss ${ }^{27}$, glow discharge plasma reduction ${ }^{32}$, and metal-block copolymer procedure $^{33}$, have been attempted to suppress the migration of metallic species. Understanding the filling, diffusion, and growth processes inside mesoporous silica during nanocasting and reduction is critical to controlled synthesis and further applications ${ }^{34-36}$

Here, we exploit an effective strategy to prevent the migration of metal species to the outside of mesoporous silica, and successfully synthesize monodispersed nanostructures with welldefined sizes and shapes, including Ag nanowires, 3D mesoporous $\mathrm{Au}, \mathrm{AuAg}$ alloys, and Pt networks, using a chemical reduction process (Fig. 1c). This strategy is based on a soft-enveloping function on the surface of the metal precursor immersed in silica and a phase transfer mechanism that occurs at the interface of the solid-liquid solution (SLS) phase (Fig. 1a). Specifically, a solvent ("liquid") that cannot dissolve metal precursors is used as a barrier layer and is designed to prevent metal species from migrating to the outside of mesoporous channels ("solid"). During the following reduction stage, a phase transfer process occurrs spontaneously across the interface of the "solution" (containing the reducing agent ions) and the "liquid" and leads to the reduction at the interface between the "solid" and "solution" phases. We believe the current methodology provides a simple and robust way to synthesize a variety of mesoporous NPs in metals.

\section{Results}

Structural characterization and controls. We chose a $3 \mathrm{D}$ mesoporous $\mathrm{Au}$ system as an example to demonstrate the validity and advantages of our method in yielding high-quality mesostructures in metals. KIT-6 (orderly mesoporous silica) was selected as a hard template to synthesize the 3D mesoporous $\mathrm{Au}$ networks. Hexane (or dichloromethane) and 1,1,3,3-tetramethyldisiloxane (TMDS) (or dimethylaminoborane (DMAB) or

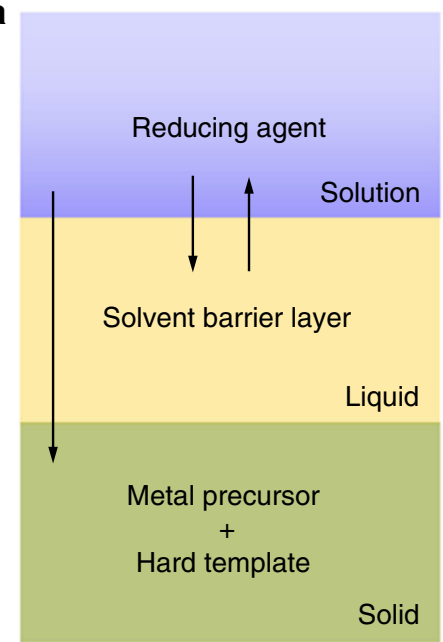

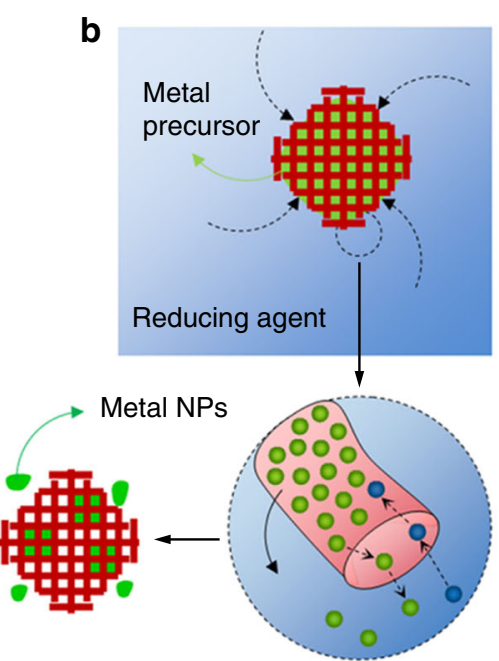

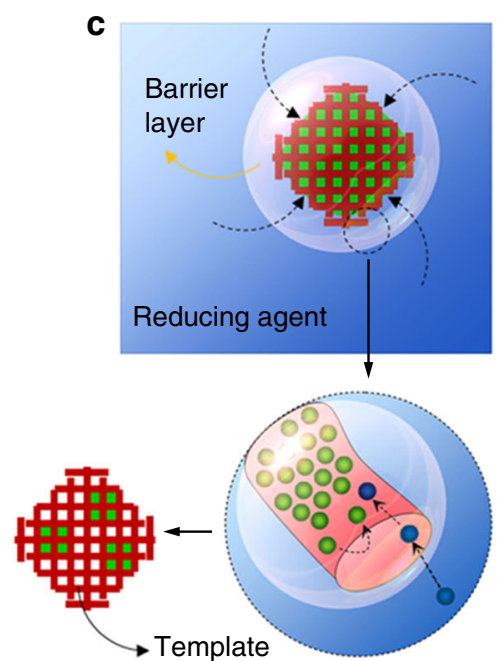

Fig. 1 The schematic illustrations of soft-enveloping strategy. a The proposed SLS (solid-liquid-solution) three phase interface, in which a solvent ("liquid") that cannot dissolve metal precursors was used as a surface coating and a barrier layer to prevent metal species from migrating to the outside of the mesoporous channels ("solid"). Next, the transfer of the reducing agent ions ("solution") across the solvent and then the reduction of the metal precursor at the interface of the "solid" and "solution". b The present challenge is that the final product mixes with the morphologies containing the bulk particles. c The current soft-enveloped SLS interface reaction protocol 

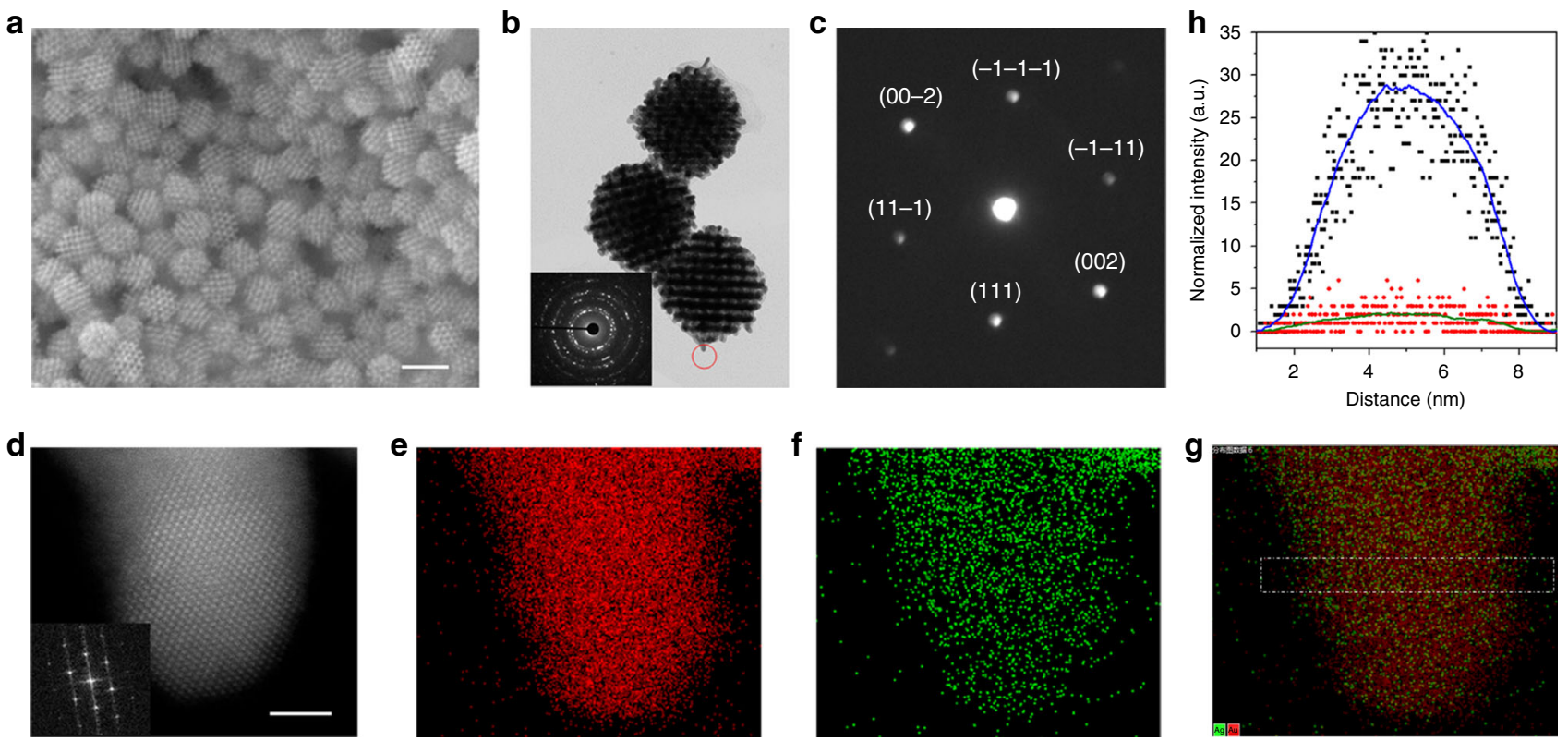

Fig. 2 The characterization analysis of the 3D ordered mesoporous AuAg networks. $\mathbf{a}, \mathbf{b}$ The typical SEM and TEM images of the 3D ordered mesoporous AuAg networked structures synthesized via the proposed soft-enveloped SLS interface reaction. c The [1-10] zone axis nanobeam electron diffraction pattern, displaying typical $\{200\}$ and $\{111\}$ Bragg reflections of fcc, Au, or Ag. d-g Representative HAADF-STEM image in combination with EDX element maps. Inset in $\mathbf{d}$ is the fast Fourier transformation (FFT) patterns of the STEM images. $\mathbf{h}$ The composition line profiles obtained by EDX with an electron beam ( $1.5 \AA$ in spot size) scanning across the region marked in $\mathbf{g}$. The scale bars in $\mathbf{a}$ and $\mathbf{d}$ are 100 and $2 \mathrm{~nm}$, respectively

butylamine) were used as the barrier layer and reducing agent, respectively. Since both hexane and dichloromethane cannot dissolve $\mathrm{HAuCl}_{4}$ and $\mathrm{AgNO}_{3}$, the reduction agent (e.g., DMAB, butylamine, or TMDS) may diffuse into the KIT-6 matrix through the barrier layer to react with $\mathrm{HAuCl}_{4}$ and $\mathrm{AgNO}_{3}$ (Fig. 1a). Thus, by means of the co-reduction of $\mathrm{HAuCl}_{4}$ and $\mathrm{AgNO}_{3}$, single component $\mathrm{Au}$ and a binary AuAg alloy can be synthesized with a $\mathrm{Ag}$ content from 0 to $12 \mathrm{wt} \%$ (determined using inductively coupled plasma atomic emission spectroscopy (ICP-AES)). Figure $2 \mathrm{a}$ shows the scanning electron microscopy (SEM) image of typical 3D mesoporous AuAg nanostructures after the removal of the silica template. The mesoporous AuAg NPs display a monodispersed ordered porous structure with good uniformity and a narrow particle size distribution with an average diameter of $\sim 100 \mathrm{~nm}$ and a standard deviation of $\sim 4.66$ (Supplementary Fig. 1). A high-magnification SEM image demonstrates that the mesoporous AuAg NPs have a polyhedral morphology, probably a cubic shape (Supplementary Fig. 2). The HRTEM images in Supplementary Fig. 3 show that large-sized mesopores approximately $14-15 \mathrm{~nm}$ are created and correspond to the total volume of the wall thicknesses plus the pore size of KIT-6. This square-shaped network structure indicates that the mesoporous AuAg NPs replicate a one-sided pore system in a bicontinuous structure ${ }^{25,37}$. Figure $2 \mathrm{~b}$ shows a transmission electron microscopy (TEM) image of the AuAg networked nanostructure. The selected-area electron diffraction (SAED) pattern inserted in Fig. $2 \mathrm{~b}$ confirms that the individual AuAg NPs are polycrystalline. While the diffraction pattern (Fig. 2c) obtained within a small region (e.g., the red circle in Fig. 2b) using a nanobeam $(\sim 1.5 \mathrm{~nm}$ in spot size) displays the typical features of a single crystal, and the reflection spots can be indexed as the Bragg reflections of a $\mathrm{Au}$ or a $\mathrm{Ag}$ face centered cubic (fcc) structure.

The phase structure and composition of the mesoporous $\mathrm{AuAg}$ NPs have been further examined at atomic level by means of aberration-corrected, high-angle annular dark-field scanning transmission electron microscopy (HAADF- STEM) in combination with energy dispersive X-ray spectroscopy (EDX), as shown in Fig. 2d-g. Figure 2d shows representative HAADFSTEM image of the mesoporous AuAg NPs. Figure $2 \mathrm{e}$ and $\mathrm{f}$ show the EDX composition maps of $\mathrm{Au}$ and $\mathrm{Ag}$, respectively, within the region of Fig. 2d. The Au (red) vs. Ag (green) composite image (Fig. 2g) displays that the $\mathrm{Au}$ and $\mathrm{Ag}$ element are highly intermixed. The sketched trend lines (Fig. 2h) obtained by scanning the e-beam across a crystal tip are almost identical across the tip, indicating the homogeneous alloy nature of the mesoporous $\mathrm{AuAg} \mathrm{NPs}{ }^{38}$. This statement is further demonstrated by a statistical analysis (Supplementary Figs. 4a-e) of the elemental distributions for different regions of the AuAg NPs and the composition line profiles obtained by EDX with an electron beam diameter of $\sim 1.5 \AA$.

Further evidence comes from X-ray photoelectron spectroscopy (XPS) data, which are shown in Supplementary Figs. 5a-c and Supplementary Figs. 6a-d. From Supplementary Fig. 5a-c, it can be observed that, compared with the standard binding energies of $\mathrm{Au}$ and $\mathrm{Ag}$, the peaks of $\mathrm{Ag} 4 \mathrm{~d}_{5 / 2}$ and $\mathrm{Ag} 4 \mathrm{~d}_{3 / 2}$ show an obvious shift. Moreover, with an increase in the Ag ratio from 0 to $12 \mathrm{wt} \%$, the $\mathrm{Au} 4 \mathrm{f}_{7 / 2}$ and $\mathrm{Au} 4 \mathrm{f}_{5 / 2}$ peaks shift to higher binding energies gradually (Supplementary Fig. 6a, b). The XPS peak shifting can be related to the perturbation in the electronic interaction between the $\mathrm{Au}$ and $\mathrm{Ag}$ atomic orbitals and in turn to alloy formation ${ }^{39}$. The X-ray diffraction (XRD) pattern of mesoporous AuAg NPs is shown Supplementary Fig. 7. The four diffraction peaks are consistent with those of $\mathrm{Au}$ or $\mathrm{Ag}$ metals with a fcc structure that correspond to the (111), (200), (220), and (311) planes. Based on the HAADF-STEM, EDX, XPS, and XRD data, the controlled phase structure of bimetallic AuAg alloy NPs with a composition of $\sim 12 \mathrm{wt} \% \mathrm{Ag}$ can be obtained by adjusting the feeding ratio of $\mathrm{AgNO}_{3}{ }^{40,41}$.

In addition to the phase structure control, the current soft-enveloping strategy also demonstrates also a robust capability in shape or morphology control as shown in Supplementary Figs. 8-10. A variety of Au nanostructures, such as NPs, dog-bone shapes, multiple pods, and mesoporous structures 

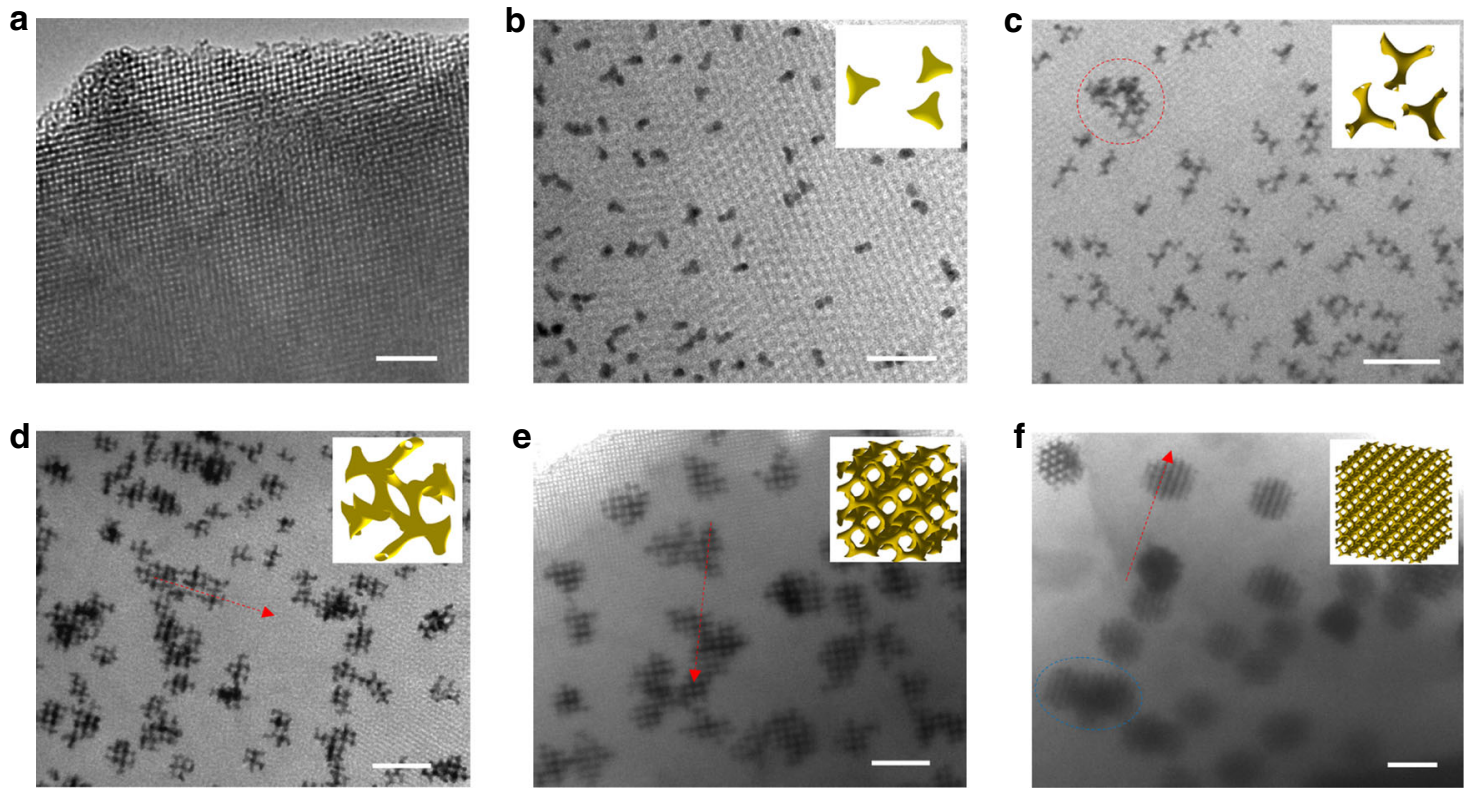

Fig. 3 TEM images of a series of Au nanostructures/silica composites. Different morphologies and sizes is obtained by changing the reduction time. a $0 \mathrm{~min}$, before the reduction reaction, displaying the Au precursor distributed homogenously in the ordered mesoporous silica matrix. $\mathbf{b} 2$ min, showing the Au nanoparticles with a wide size distribution. c 8 min, the Au NPs show a structure of three-pods. d 15 min, the Au NPs with networked morphology has been formed. e-f 2 and $6 \mathrm{~h}, 3 \mathrm{D}$ mesoporous Au networks, the red arrows indicate that the Au NPs deposited within the same single mesostructural la3d domain of the original KIT-6. The scale bars in $\mathbf{a}, \mathbf{b}$, and $\mathbf{c}-\mathbf{f}$ are 100,50 , and $100 \mathrm{~nm}$, respectively

(Supplementary Figs. 8-19) with tunable sizes can be successfully synthesized by controlling the growth time of the formation of the Au structure. Supplementary Figs. 8a-f show the schematic illustration for the synthesis of well-defined nanostructured $\mathrm{Au}$ NPs from a KIT-6 silica template. During the early stage of Au growth within $10 \mathrm{~min}$, spherical Au NPs, dog-bone shapes, and multiple pods are obtained (Supplementary Figs. 9a and 8a). With an increase in the reduction time from $30 \mathrm{~min}$ to $24 \mathrm{~h}$, the $\mathrm{Au}$ NPs display an ordered mesoporous morphology with increasing sizes from $\sim 50 \mathrm{~nm}$ (Supplementary Figs. $8 \mathrm{c}$ and $9 \mathrm{~b}$ ) to $\sim 100 \mathrm{~nm}$ (Supplementary Fig. 9c) and $\sim 150 \mathrm{~nm}$ (Supplementary Fig. 9d). To investigate the morphologies of the products under a variety of reaction conditions, we have also studied the influence of reactant concentrations. At relatively low or high $\mathrm{HAuCl}_{4}$ concentrations, e.g., $0.5,10$, and $100 \mathrm{mM}$, ordered mesoporous $\mathrm{Au}$ nanostructures with different particle sizes can still be obtained (Supplementary Figs. 10a-c). It is revealed that the current synthetic strategy has a strong capability to tune the morphologies and sizes of the products.

Formation mechanism. To understand the growth processes of mesoporous Au NPs within a KIT-6 matrix, the mesoporous Au/ silica composites before silica removal were carefully investigated by TEM. Figure 3 demonstrates a series of $\mathrm{Au}$ nanostructures/ silica composites with different morphologies and sizes prepared by changing the reduction time, i.e., $0,2,8,15 \mathrm{~min}, 2$ and $6 \mathrm{~h}$. Initially, the $\mathrm{Au}$ precursor is distributed homogenously in the ordered mesoporous silica matrix (Fig. 3a and Supplementary Fig. 11). During the very early stage of the reduction reaction, several $\mathrm{Au}$ NPs with sizes from less than 10 to $\sim 20 \mathrm{~nm}$ are observed clearly (Fig. 3b and Supplementary Fig. 12). During the subsequent growth, three pods or multiple pods and even some network clusters (circled in red in Fig. 3c) can be formed. In this situation, the sizes of multiple pods and clusters are still distributed in a wide range of $20-50 \mathrm{~nm}$. As the reaction proceeds, i.e., after $15 \mathrm{~min}$, small-sized mesoporous $\mathrm{Au}$ networks are obtained, displaying a size of approximately 50-70 nm (Fig. 3d). When the reduction time approaches to 2 and $6 \mathrm{~h}$ (Fig. $3 \mathrm{e}, \mathrm{f}$, respectively), the morphologies of the mesoporous $\mathrm{Au}$ networks are not changed, but the average particle sizes reach approximately 80 and $100 \mathrm{~nm}$, respectively. Based on the further observations in Fig. 3, the Au NPs during the early stage are distributed over the entire silica matrix and isolated from each other. The initially formed Au networks (Fig. 3d) displayed a '\#'-like structural feature, which contributes to the final formation of polyhedral shapes (Fig. 3e, f). In addition, during the growth of the mesoporous Au networks, the Au NPs are deposited within the same single mesostructural Ia3d domain of the original KIT-6 (red arrows in Fig. 3e, f) materials, revealing that a well-controlled replication process of the mesoporous silica has been successfully realized.

According to the above results, the growth mechanism of the 3D mesoporous Au network is proposed and schematically illustrated in Supplementary Fig. 13. The formation of mesoporous $\mathrm{Au}$ networks that originate from homogenously distributed Au precursors within a limited space (mesoporous channels) can be described by the classical LaMer curve ${ }^{42}$. These networks consist of three distinct periods: (i) an induction period, (ii) a nucleation period, and (iii) a growth period of Au multiple pods and the mesoporous network. During the induction period, $\mathrm{Au}^{3+}$ ions are reduced to $\mathrm{Au}^{0}$ atoms. According to time-dependent growth feature of the Au networked nanostructures as shown in Fig. 3 and Supplementary Figs. 9 and 10, the reduced Au atoms are uniformly distributed over the entire silica matrix ${ }^{25}$. Following the induction period, $\mathrm{Au}$ atoms start to nucleate via a self- (or homogeneous) nucleation process once the concentration of $\mathrm{Au}$ atoms exceeds the supersaturation point. After the nucleation period, according to the in situ UV spectral measurements (Supplementary Figs. 14a and b) and the uniform particle size features shown in Fig. 3, no additional "secondary nucleation" can occur, and most of the reaction time is spent during the growth process ${ }^{25}$. These statements are nicely supported by the following: (i) The density of the Au NPs, 

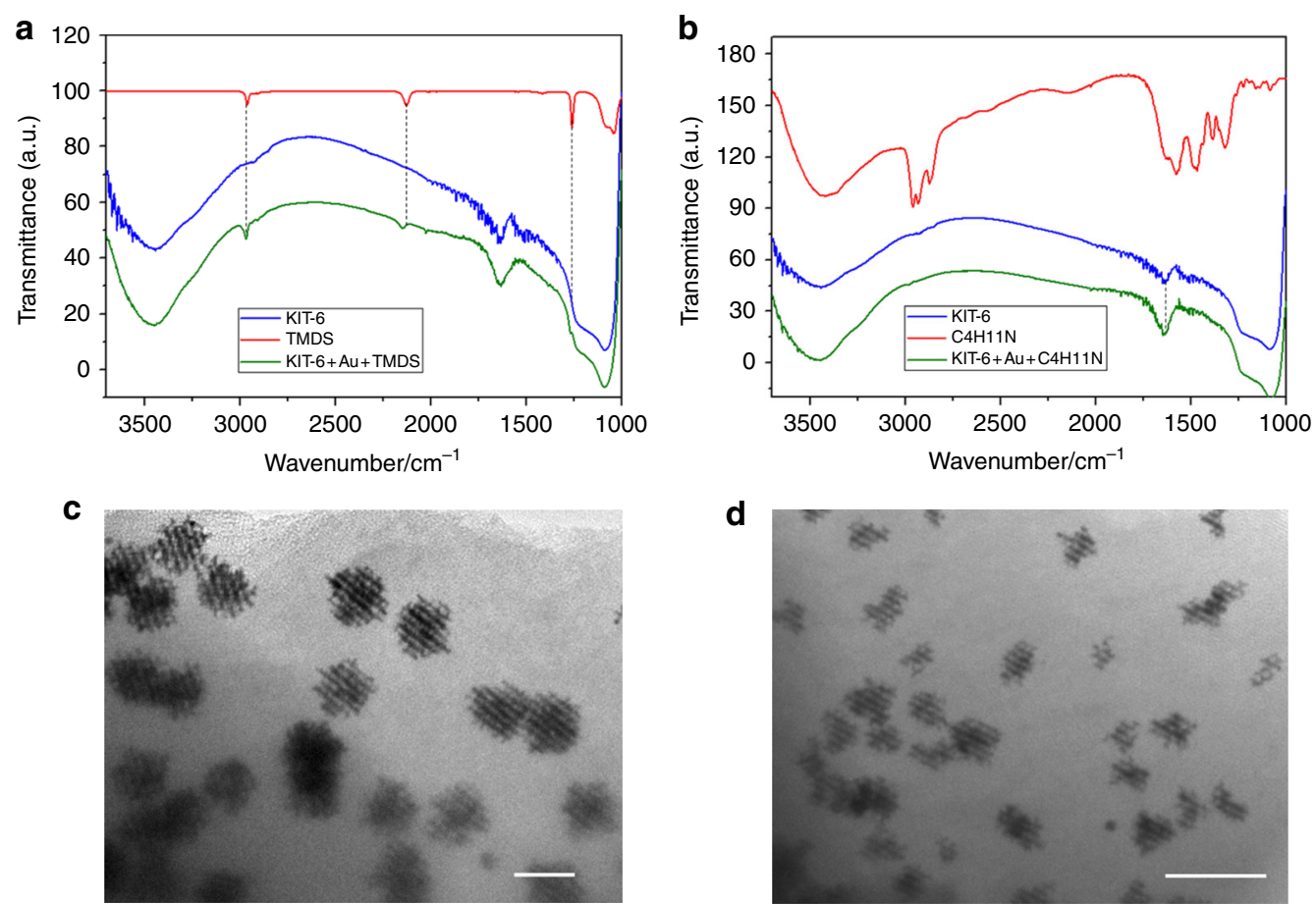

Fig. 4 The influences of the reduction agent and solvent barrier layer. a FT-IR spectra of the KIT-6 mesoporous template, TMDS reduction agent, and Au compounds. b FT-IR spectra of the KIT-6 mesoporous template, butylamine reduction agent, and Au compounds. c 3D mesoporous Au networks/silica compounds using butylamine as the reduction agent and hexane as the solvent (scale bar, $100 \mathrm{~nm}$ ). d 3D mesoporous Au networks/silica compounds using TMDS as the reduction agent and dichloromethane as the solvent (scale bar, $200 \mathrm{~nm}$ )

multiple pods and mesoporous networks (i.e., the number of $\mathrm{Au}$ particles per unit area) do not increase as the growth time is increased (Fig. 3). The slight discrepancy of density may be caused by a bias in the supply of Au sources among the silica matrix. (ii) No small Au clusters can be observed near the mesoporous Au networked NPs (Fig. 3). (iii) The in situ UV spectroscopy measurements can also reveal that, after a very early stage within $60 \mathrm{~s}$, the Au cluster peaks at approximately $400 \mathrm{~nm}$ do not appear again during the following growth period (Supplementary Fig. 14b), indicating that no new clusters or nuclei can be continuously formed ${ }^{43,44}$. During the growth period, the $\mathrm{Au}$ ions and atoms diffuse from the associated space to each nucleus (i.e., the Voronoi cell) ${ }^{25}$ by tracing the channels of the KIT- 6 template, and finally contribute to the growth of the Au nanostructures, including multiple pods and mesoporous network morphologies. In this case, the formed instable nuclei may dissolve into the solution and support the growth process again; thus resulting in the formation of a mesoporous Au networked structure with uniform sizes. In addition, since we are not equipped with a real-time TEM observation capability, we cannot exclude the importance of the aggregation of small NPs (e.g., less than the diameter of the KIT- 6 channels). While we believe in present system, the growth of Au networks is an atom-mediated process.

In the current soft-enveloping synthetic strategy, hexane and TMDS are mainly selected as the barrier layer solvent and reduction agent, respectively, to avoid the migration of metal species to the outside of the mesoporous silica. In the absence of a barrier layer ("liquid") in the synthetic sysem (Fig. 1a), some bulk particles without an ordered mesoporous structure can obviously be observed because of $\mathrm{Au}$ growth on the outer surface of the mesoporous silica template, as shown in the TEM (Supplementary Fig. 15a) and SEM images (Supplementary Fig. 16a). However, with a barrier layer, the Au species can be effectively controlled to grow inside the mesoporous silica (Supplementary
Figs. $15 \mathrm{~b}$ and $16 \mathrm{~b})$. The non-polar TMDS reducing agent may react with the polar silica surfaces and hence suppress the migration of the Au species. This reaction is supported by the FT-IR spectra shown in Fig. 4a, where the peaks located at $2129 \mathrm{~cm}^{-1}$ (Si-H stretching) and $2961 \mathrm{~cm}^{-1}\left(\mathrm{CH}_{3}\right.$ symstretching) are observed after the reaction. However, the further comparison experiments indicate that the soft-enveloping barrier layer may also play a crucial role to prevent metal species from migrating to the outside of the mesoporous channels. If TMDS is replaced by other reduction agents (e.g., non-polar dimethylaminoborane (DMAB) or polar butylamine), or the soft-enveloping solvent layer was replaced by other solvents (e.g., dichloromethane), then the networked $\mathrm{Au}$ nanostructures can be obtained and no obvious bulk particles that have grown outside of silica channels can be observed (Fig. 4c, d and Supplementary Figs. 17-19). Particularly, the FT-IR spectra indicate that the polar butylamine reduction agent does not demonstrate a remarkable reaction between the silica surface and butylamine (Fig. 4b). However, in this system, the well-controlled $\mathrm{Au}$ network may still be obtained, further supporting the validity of the current soft-enveloping synthetic strategy.

Catalytic property. Because of the novel 3D framework structure, the high specific surface area, high internal porosity, and rough surface of the mesoporous $\mathrm{Au}$ networked nanostructures demonstrate diverse outstanding properties that can be utilized in spectral signal enhancement, controlled drug delivery and cancer combination therapy, and catalysis. To evaluate the catalytic activity of the material, we measured the electrochemical oxidation of methanol as a model reaction for the $3 \mathrm{D}$ mesoporous $\mathrm{Au}$ and AuAg networks (Fig. 5). For comparison, six kinds of Au NPs with diameters of approximately 3, 4, 5, 6, 20, and $70 \mathrm{~nm}$ (as shown in Supplementary Fig. 20a-f and Supplementary Fig. 21), were synthesized and investigated. As shown in Supplementary 

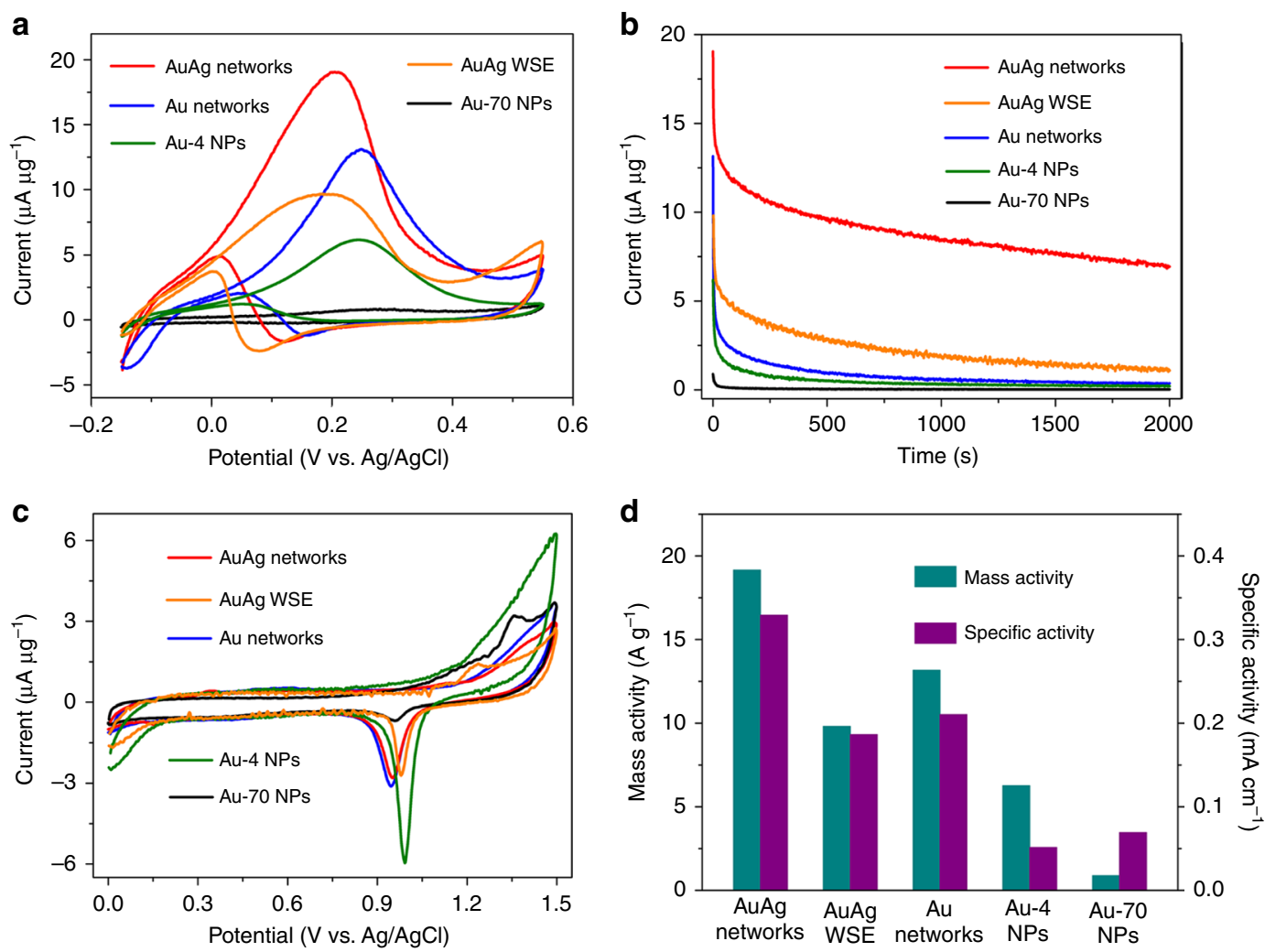

Fig. 5 The electrochemical performance. a Cyclic voltammograms of the electro-methanol oxidation, $\mathbf{b}$ chronamperograms, c oxide stripping curves, and d histograms of the mass activity and specific activity of the AuAg networks, Au networks, Au-4 NPs, and Au-70 NPs. The cyclic voltammograms were performed in $0.5 \mathrm{M} \mathrm{KOH}$ and $2 \mathrm{M} \mathrm{CH}_{3} \mathrm{OH}$ at a scan rate of $10 \mathrm{mV} \mathrm{s}^{-1}$. The oxide stripping was measured in a $0.5 \mathrm{M} \mathrm{H}_{2} \mathrm{SO}_{4}$ solution at a scan rate of $10 \mathrm{mV} \mathrm{s}$

Fig. 22a-c, among these Au NPs, $4 \mathrm{~nm}$-sized Au NPs show a better mass activity, and the $70 \mathrm{~nm}$-sized Au NPs display a better specific activity. Thus, $4 \mathrm{~nm}$ and $70 \mathrm{~nm} \mathrm{Au}$ nanoparticles (denoted as Au-4 and Au-70 NPs) are selected as references in the study of the $\mathrm{Au}$ and AuAg network catalytic property for the methanol oxidation reaction (MOR). In addition, the catalytic property of AuAg products (Supplementary Fig. 15a) synthesized without the soft-enveloping strategy (denoted as AuAg WSE) was also measured as a comparison.

From Fig. 5a, one can see that Au-4 NPs and Au-70 NPs exhibit peak currents for methanol oxidation at 6.2 and $0.9 \mu \mathrm{A} \mu \mathrm{g}^{-1}$, respectively. While the AuAg networks show much higher peak

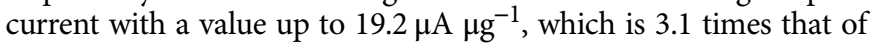
Au-4 NPs, and 24 times that of Au-70 NPs. Moreover, the peak potentials for methanol oxidation are $0.20,0.20,0.25,0.26$, and $0.27 \mathrm{~V}$ (Fig. 5a), revealing that methanol oxidation can be easier with the AuAg, and Au networked catalysts and exhibit lower electro-oxidation potentials than those of the Au- 4 and Au-70 NPs. The rate of surface poisoning for the five catalysts was evaluated and shown in Fig. 5b. As in previous reports ${ }^{45,46}$, the lagging decreasing rate and the highest current during the entire chronoamperometry process ( $2000 \mathrm{~s}$ ) are observed for the AuAg networked catalysts, indicating that the electrocatalytic stability of the AuAg networks for the methanol oxidation reaction (MOR) is higher than that of the AuAg WSE, Au networks, Au-4 NPs, and Au-70 NPs. Considering the electrochemically active surface areas (ECSAs) (Fig. 5c) and the mass, both the Au and AuAg networks show greatly improved specific activity and mass activity (Fig. 5d). Specifically, the AuAg networks possess a very high catalytic activity, which is 7 and 5 times higher in specific activity and 3 and 24 times higher in mass activity than that of the $\mathrm{Au}-4$ and $\mathrm{Au}-70$ NPs, respectively (Fig. 5d). Table 1 summarizes the specific activities for several $\mathrm{Au}$ and $\mathrm{Au}$ alloy catalysts reported in the literature ${ }^{13,47-53}$. Different scanning rates have been used in different studies, and normally a higher current can be obtained with a higher scan rate ${ }^{54}$. Thus, considering the test conditions (i.e., lower scan rate, $10 \mathrm{mV} \mathrm{s}^{-1}$, was used in our measurements), our AuAg alloy networks show greatly improved catalytic property compared with that of the Au or AuAg catalysts reported in the literature. In addition to the high catalytic activity and stability, the AuAg networks also show a greatly enhanced durability compared with that of the Au NPs (Supplementary Figs. 23a-c and Supplementary Discussion).

Referring to Fujita's group and Ling's group reports ${ }^{9,13}$, we may attribute the superior catalytic performance of the AuAg networks to the high density of the low coordinated atoms, atomic steps and kinks on the curved surface of networked NPs, and the high-index facets (Supplementary Fig. 27), which are formed by the limited growth in the narrow spaces of the mesoporous template. In fact, the obtained atomic structure of the AuAg networks is quite similar to the structure of nanoporous gold prepared via a dealloying process ${ }^{13}$. A more detailed discussion regarding catalytic properties can be seen in the Supplementary Information (Supplementary Figs. 24-26 and Supplementary Discussion).

Generalization of the current strategy in other systems. The current synthetic strategy can effectively avoid diffusion of metal species to the outside of the mesoporous channels; thus, metallic nanostructures within the mesoporous templates are obtained with controlled shape or morphology. In fact, this protocol may also be easily extended to rather general systems. As mentioned above, we have obtained similar Au mesoporous structures with 
Table 1 Specific activity of the Au and AuAg catalysts for the MOR reported in the literature

\begin{tabular}{|c|c|c|c|}
\hline Types of Au or AuAg catalysts & Measurement conditions $^{a}$ & Specific activity ${ }^{b}$ & Ref. \\
\hline AuNPs/LDH/GC electrode & $50,0.5,0.25$ & 0.11 & {$[48]$} \\
\hline Au NPs on vitreous carbon & $50,0.1,0.1$ & 0.12 & [49] \\
\hline Mesoporous Au film & $20,2,0.1$ & 0.2 & {$[51]$} \\
\hline De-alloyed Au NFs & $10,1,0.1$ & 0.25 & {$[52]$} \\
\hline Nanoporous gold & $10,2,0.5$ & 0.09 & {$[53]$} \\
\hline
\end{tabular}
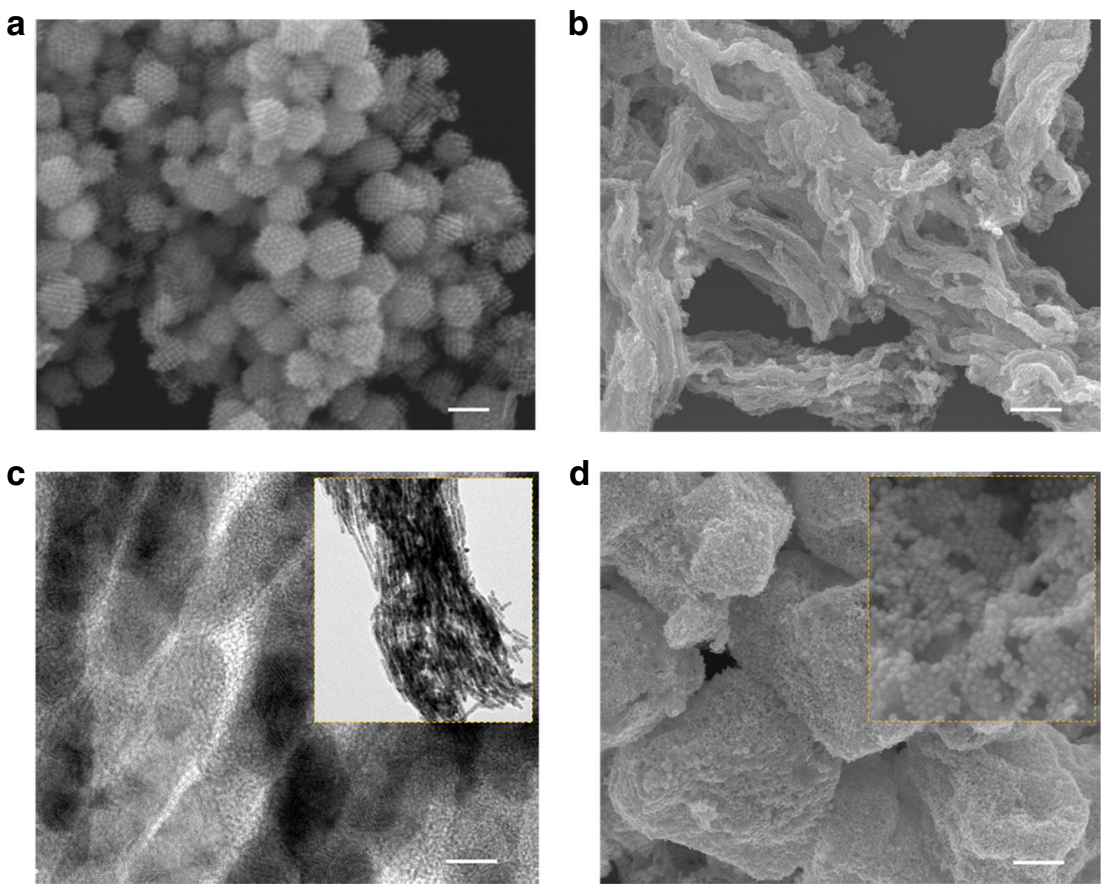

Fig. 6 The effectiveness proof of the SLS strategy in other systems. a The 3D mesoporous Pt networked structure prepared by using a KIT-6 template. b,c Ag nanowires obtained via an SBA-15 template. $\mathbf{d}$ The 3D mesoporous Au nanoparticle superlattice prepared using EP-FDU-12 as the template. The insets in $\mathbf{c}$ and $\mathbf{d}$ are the magnified images of corresponding structures obtained from the current soft-enveloped SLS interface reaction strategy. The scale bars in $\mathbf{a}, \mathbf{b}, \mathbf{c}$, and $\mathbf{d}$ are $100,500,5$, and $500 \mathrm{~nm}$, respectively

well-controlled morphology by means of various reduction agents such as TMDS, DMAB and butylamine, and barrier layer solvents, such as hexane and dichloromethane. To evaluate the validity of our understanding and the generality of the softenveloping method, we further applied this method to another replica system.

As shown in Fig. 6, three kinds of $\mathrm{Pt}, \mathrm{Au}$ and $\mathrm{Ag}$ nanostructures are obtained from the current soft-enveloped SLS interface reaction strategy by means of a similar synthetic approach but with changes only to the metal species and templates. Using a KIT-6 mesoporous silica template, the 3D mesoporous Pt networked structure can be prepared (Fig. 6a). Similarly, 1D Ag nanowires, and Au nanoparticle superlattices may also be fabricated using the SBA-15 and EP-FDU-12 mesoporous templates, as shown in Fig. 6b-d. Note that in all the reaction systems (Fig. 6), the products demonstrate a single morphological feature, revealing the deposition of metal nanostructures outside the mesoporous silica template has been effectively avoided. As a comparison, we have also evaluated the validity of the current protocol by removing the solvent barrier layer. It was clearly seen that, when the synthetic system was absent of the barrier layer, bulk NPs can always observed in all three above reaction systems as a result of metal growth on the outer surface of the mesoporous silica template (Supplementary Figs. 28a-c).

\section{Discussion}

In this work, we have proposed a robust synthetic strategy via a general soft-enveloping SLS interface reaction to effectively prevent metal species from migrating to the outside of mesoporous channels. Au or AuAg alloy nanostructures were successfully obtained with diverse morphologies, including spherical NPs, multiple pods, and networks. The 3D mesoporous AuAg networked structure demonstrates outstanding electrocatalytic properties that include enhancements in specific activity and mass activity of 7 and 3 times, respectively, over $4 \mathrm{~nm}$-sized Au NPs. By extending the soft-enveloped SLS interface reaction 
strategy to other replica systems using different barrier layer solvents, reduction agents, metal species and mesoporous templates, a variety of mesostructures have also been synthesized, including the 3D mesoporous Pt networks, 1D Ag nanowires, and $\mathrm{Au}$ nanoparticle superlattices. Finally, our strategy and results offer a synthetic platform to generate a new class of mesoporous metal nanostructures, thus opening avenues to exploit diverse applications in catalysis, optics and biomedicine ${ }^{55}$.

\section{Methods}

Synthesis of mesoporous Au networks. In a typical impregnation process, the dried KIT-6 (0.1 g) powder was immersed into ethanol solution of $\mathrm{HAuCl}_{4}(3 \mathrm{mM}$, $10 \mathrm{ml}$ ). Then, the mixed solution was dried under reduced vacuum condition to incorporate the Au precursor into the mesopores. After the complete drying, yellow-colored powder was obtained. Then, the powder was dispersed in hexane (1 $\mathrm{ml})$, TMDS $(100 \mu \mathrm{l})$ was added into the hexane solution. The Au deposition was carried out in a closed vessel for various periods while the color was changed to black. The obtained power was washed with hexane and ethanol, and dried in room temperature. The silica template was removed by hydrofluoric acid (HF, $20 \mathrm{wt} \%$ ). Black powder were centrifuged and washed with distilled water and ethanol, then dried up at room temperature.

Characterization. The morphology and structure of the product were characterized using a scanning electron microscope (SEM, JEOL, JSM-7000F) and a transmission electron microscope (TEM, JEOL, JEM-2100 with an accelerating voltage of $200 \mathrm{kV}$ ). The HAADF-STEM image and energy-dispersive spectroscopy elemental mapping of the product were obtained by scanning transmission electron microscopy (STEM, JEOL, JEM-ARM 200F). The chemical composition of the product was characterized using X-ray diffraction (XRD, Bruker), X-ray photoelectron spectroscopy (XPS, Thermo Fisher Scientific, ESCALAB 250Xi+) and Inductive Coupled Plasma Emission Spectrometer (ICP, SHIMADZU, ICPE-9000). The In situ UV spectroscopy measurements were characterized using Ultraviolet-visible spectroscopy (UV, Shimadzu, UV-60).

Electrochemical measurements. The electrochemical measurement was performed on a VersaSTAT 3 electrochemical working station using a three-electrode cell. The working electrode was a glassy-carbon rotating disk electrode (GCE, $5 \mathrm{~mm}$ in diameter). A platinum foil with area of $1 \mathrm{~cm}^{2}$ was used as the counter electrode, and a double junction $\mathrm{Ag} / \mathrm{AgCl}$ electrode was used as the reference. Before the measurement for the catalytic property, the catalyst was firstly treated by 30 cycles of $\mathrm{CV}$ (between 0.3 and $0.7 \mathrm{~V}$ ) in $0.5 \mathrm{M} \mathrm{KOH}$ aqueous solution with a scan rate of $50 \mathrm{mV} \mathrm{s}^{-1}$ to remove the remaining of $\mathrm{SiO}_{2}$. The samples for the measurement of $\mathrm{Au}$ nanoparticles were not treated with $\mathrm{KOH}$ solutions. Then, the measurement for the catalytic property was performed in deoxygenated solution of $0.5 \mathrm{M} \mathrm{KOH}$ and $2 \mathrm{M}$ methanol with a scan rate of $10 \mathrm{mV} \mathrm{s}^{-1}$. The active surface area of various catalysts was estimated using the $\mathrm{CV}$ curve measured in deoxygenated $0.5 \mathrm{M}$ $\mathrm{H}_{2} \mathrm{SO}_{4}$ aqueous solution at a scan rate of $10 \mathrm{mV} \mathrm{s}^{-1}$. The accelerated durability test (ADT) was performed using $\mathrm{CV}$ cycles in $\mathrm{O}_{2}$ saturated $0.5 \mathrm{M} \mathrm{KOH}+2 \mathrm{M} \mathrm{CH}_{3} \mathrm{OH}$ aqueous solution with scanningfrom -0.15 to $0.55 \mathrm{~V}$ (vs Ag/AgCl) at rate of $10 \mathrm{mV} \mathrm{s}^{-1}$. In order to avoid the effect of the decrease of methanol concentration on the results, very 500 cycles, new prepared solution is used to complete the ADT.

Data availability. The authors declare that all the data supporting the findings of this study are available within the paper and its Supplementary Information or from the corresponding author upon reasonable request.

Received: 14 March 2017 Accepted: 9 January 2018

Published online: 06 February 2018

\section{References}

1. Kresge, C. T., Leonowicz, M. E., Roth, W. J., Vartuli, J. C. \& Beck, J. S. Ordered mesoporous molecular sieves synthesized by a liquid-crystal template mechanism. Nature 359, 710-712 (1992).

2. Zhao, D. Y. et al. Triblock copolymer syntheses of mesoporous silica with periodic 50 to 300 angstrom pores. Science 279, 548-552 (1998).

3. Yang, P. D., Zhao, D. Y., Margolese, D. I., Chmelka, B. F. \& Stucky, G. D. Generalized syntheses of large-pore mesoporous metal oxides with semicrystalline frameworks. Nature 396, 152-155 (1998).

4. Yang, Y. N. et al. Synthesis of SBA-15 rods with small sizes for enhanced cellular uptake. J. Mater. Chem. B 2, 4929-4934 (2014).

5. Jambhrunkar, S. et al. Stepwise pore size reduction of ordered nanoporous silica materials at angstrom precision. J. Am. Chem. Soc. 135, 8444-8447 (2013).
6. Song, H. et al. Silica nanopollens enhance adhesion for long-term bacterial inhibition. J. Am. Chem. Soc. 138, 6455-6462 (2016).

7. Tian, C. F., Deng, Y. H., Zhao, D. Y. \& Fang, J. X. Plasmonic silver supercrystals with ultrasmall nanogaps for ultrasensitive SERS-based molecule detection. Adv. Opt. Mater. 3, 404-411 (2015).

8. Ma, C., Gao, Q. Q., Hong, W., Fan, J. \& Fang, J. X. Real-time probing nanopore-in-nanogap plasmonic coupling effect on silver supercrystals with surface-enhanced Raman spectroscopy. Adv. Funct. Mater. 27, 1603233 (2017).

9. Fujita, T. et al. Atomic origins of the high catalytic activity of nanoporous gold. Nat. Mater. 11, 775-780 (2012)

10. Wittstock, A., Zielasek, V., Binner, J., Friend, C. M. \& Baumer, M. Nanoporous gold catalysts for selective gas-phase oxidative coupling of methanol at low temperature. Science 327, 319-322 (2010).

11. Li, C. L. et al. Electrochemical synthesis of mesoporous gold films toward mesospace-stimulated optical properties. Nat. Commun. 6, 6608-6614 (2015).

12. Tian, C. F. et al. Ordered mesoporous Ag superstructure synthesized via template strategy for surface-enhanced Raman scattering. Nanoscale 7, 12318-12324 (2015)

13. Pedireddy, S. et al. One-step synthesis of zero-dimensinal hollow nanoporous gold nanoparticles with enhanced methanol electrooxidation performance. Nat. Commun. 5, 4947-4956 (2014).

14. Han, Y. J., Kim, J. M. \& Stucky, G. D. Preparation of noble metal nanowires using hexagonal mesoporous silica SBA-15. Chem. Mater. 12, 2068-2069 (2000).

15. Kang, H. Y., Jun, Y. W., Park, J. I., Lee, K. B. \& Cheon, J. Synthesis of porous palladium superlattice nanoballs and nanowires. Chem. Mater. 12, 3530-3532 (2000).

16. Kanno, Y., Suzuki, T., Yamauchi, Y. \& Kuroda, K. Preparation of Au nanowire films by electrodeposition using mesoporous silica films as a template: vital effect of vertically oriented mesopores on a substrate. J. Phys. Chem. C 116, 24672-24680 (2012).

17. Ruan, Q. F., Shao, L., Shu, Y. W., Wang, J. F. \& Wu, H. K. Growth of monodisperse gold nanospheres with diameters from $20 \mathrm{~nm}$ to $220 \mathrm{~nm}$ and their core/satellite nanostructures. Adv. Opt. Mater. 2, 65-73 (2014).

18. Song, G. S. et al. Degradable molybdenum oxide nanosheets with rapid clearance and efficient tumor homing capabilities as a therapeutic nanoplatform. Angew. Chem. Int. Ed. 55, 2122-2126 (2016).

19. Song, G. S. et al. Perfluorocarbon-loaded hollow $\mathrm{Bi}_{2} \mathrm{Se}_{3}$ nanoparticles for timely supply of oxygen under near-infrared light to enhance the radiotherapy of cancer. Adv. Mater. 28, 2716-2723 (2016).

20. Chen, Q., Liang, C., Wang, C. \& Liu, Z. An imagable and photothemal "abraxane-like" nanodrug for combination cancer therapy to treat subcutaneous and metastatic breast tumors. Adv. Mater. 27, 903-910 (2015).

21. Li, W., Liu, J. \& Zhao, D. Y. Mesoporous materials for energy conversion and storage devices. Nat. Rev. Mater. 1, 16023-16040 (2016).

22. Shon, J. K. et al. Facile synthesis of highly ordered mesoporous silver using cubic mesoporous silica template with controlled surface hydrophobicity. Chem. Commun. https://doi.org/10.1039/B811718G (2009).

23. Zhang, F. et al. Fabrication of $\mathrm{Ag} @ \mathrm{SiO}_{2} @ \mathrm{Y}_{2} \mathrm{O}_{3}$ : Er nanostructures for bioimaging: tuning of the upconversion fluorescence with silver nanparticles. J. Am. Chem. Soc. 132, 2850-2859 (2010).

24. Shin, H. J., Ryoo, R., Liu, Z. \& Terasaki, O. Template synthesis of asymmetrically mesostructured platinum networks. J. Am. Chem. Soc. 123 1246-1247 (2001)

25. Wang, H. J. et al. Shape- and size- controlled synthesis in hard templates: sophisticated chemical reduction for mesoporous monocrystalline platinum nanoparticles. J. Am. Chem. Soc. 133, 14526-14529 (2011).

26. Li, Z., Kubel, C., Parvulescu, V. I. \& Richards, R. Size tunable gold nanorods evenly distributed in the channels of mesoporous silica. ACS Nano 2, 1205-1212 (2008)

27. Kitahara, M. \& Kuroda, K. Preparation of highly controlled nanostructured $\mathrm{Au}$ within mesopores using reductive deposition in non-polar environments. RSC Adv. 4, 27201-27206 (2014).

28. Kuroda, Y. \& Kuroda, K. Morphosynthesis of nanostructured gold crystals by utilizing interstices in periodically arranged silica nanoparticles as a flexible reaction field. Angew. Chem. Int. Ed. 49, 6993-6997 (2010).

29. Takai, A., Doi, Y., Yamauchi, Y. \& Kuroda, K. Soft-chemical approach of noble metal nanowires templated from mesoporous silica (SBA-15) through vapor infiltration of a reducing agent. J. Phys. Chem. C 114 7586-7593 (2010)

30. Besson, S., Gacoin, T., Ricolleau, C. \& Boilot, J. Silver nanoparticle growth in 3D-hexagonal mesoporous silica films. Chem. Commun. https://doi.org/ 10.1039/B208357D (2003)

31. Plyuto, Y., Berquier, J. M., Jacquiod, C. \& Ricolleau, C. Ag nanoparticles synthesized in template-structured mesoporous silica films on a glass substrate. Chem. Commun. https://doi.org/10.1039/A904681J (1999). 
32. Wang, Z. J., Xie, Y. \& Liu, C. J. Synthesis and characterization of noble metal $(\mathrm{Pd}, \mathrm{Pt}, \mathrm{Au}, \mathrm{Ag})$ nanostructured materials confined in the channels of mesoporous SBA-15. J. Phys. Chem. C 112, 19818-19824 (2008).

33. Zhu, J. et al. Encapsulation of metal ( $\mathrm{Au}, \mathrm{Ag}, \mathrm{Pt})$ nanoparticles ino the mesoporous SBA-15 structures. Langmuir 19, 4396-4401 (2003).

34. Sun, X. H. et al. Container effect in nanocasting synthesis of mesoporous metal oxides. J. Am. Chem. Soc. 133, 14542-14545 (2011).

35. Yang, C. M., Sheu, H. S. \& Chao, K. J. Templated synthesis and structural study of densely packed metal nanostructures in MCM-41 and MCM-48. Adv. Funct. Mater. 12, 143-148 (2002).

36. Warren, S. C. et al. Ordered mesoporous materials from metal nanoparticleblock copolymer self-assembly. Science 320, 1748-1752 (2008).

37. Doi, Y. et al. Tailored synthesis of mesoporous platinum replicas using double gyroid mesoporous silica (KIT-6) with different pore diameters via vapor infiltration of a reducing agent. Chem. Commun. 46, 6365-6367 (2010).

38. Liu, Z. et al. Highly sensitive, uniform, and reproducible surface-enhanced Raman spectroscopy from hollow Au-Ag alloy nanourchins. Adv. Mater. 26, 2431-2439 (2014).

39. Xu, J. B., Zhao, T. S., Yang, W. W. \& Shen, S. Y. Effect of surface composition of Pt-Au alloy cathode catalyst on the performance of direct methanol fuel cells. Int. J. Hydrog. Energy 35, 8699-8706 (2010).

40. Zhang, Q. F. et al. Intertwining roles of silver ions, surfactants, and reducing agents in gold nanorod overgrowth: pathway switch between silver underpotential deposition and gold-silver codeposition. Chem. Mater. 28, 2728-2741 (2016).

41. Wang, C. et al. One-pot synthesis of oleylamine coated AuAg alloy NPs and their catalysis for CO oxidation. Chem. Mater. 21, 433-435 (2009)

42. LaMer, V. K. \& Dinegar, R. H. Theory, production and mechanism of formation of monodispersed hydrosols. J. Am. Chem. Soc. 72, 4847-4854 (1950).

43. Philip, R., Chantharasupawong, P., Qian, H. F., Jin, R. C. \& Thomas, J. Evolution of nonlinear optical properties: from gold atomic clusters to plasmonic nanocrystals. Nano Lett. 12, 4661-4667 (2012).

44. Paramanik, B. \& Patra, A. Fluorescent AuAg alloy clusters: synthesis and SERS applications. J. Mater. Chem. C 2, 3005-3012 (2014).

45. Wang, L., Nemoto, Y. \& Yamauchi, Y. Direct synthesis of spatially-controlled Pt-on-Pd bimetallic nanodendrites with superior electrocatalytic activity. $J$. Am. Chem. Soc. 133, 9674-9677 (2011).

46. Li, T., You, H. J., Xu, M. W., Song, X. P. \& Fang, J. X. Electrocatalytic properties of hollow coral-like platinum mesocrystals. ACS Appl. Mater. Interfaces 4, 6941-6947 (2012).

47. Wang, Y. L., Zhang, D. D., Tang, M., Xu, S. D. \& Li, M. G. Electrocatalysis of gold nanoparticles/layered double hydroxides nanocomposites toward methanol electro-oxidation in alkaline medium. Electrochim. Acta 55, 4045-4049 (2010).

48. Hernandez, J., Solla-Gullon, J., Herrero, E., Aldaz, A. \& Feliu, J. M. Methanol oxidation on gold nanoparticles in alkaline media: unusual electrocatalytic activity. Electrochim. Acta 52, 1662-1669 (2006).

49. Sun, Y., Sang, J. L., Wang, X. \& Li, Y. J. Electrochemical fabrication of threedimensional fern-like Au nanostructure and its electrocatalytic activity towards methanol oxidation. Electrochim. Acta 216, 88-93 (2016).

50. Li, C. L. et al. Superior electrocatalytic activity of mesoporous Au film templated from diblock copolymer micelles. Nano Res 9, 1752-1762 (2016).
51. Li, G. G. et al. Controlled dealloying of alloy nanoparticles toward optimization of electrocatalysis on spongy metallic nanoframes. ACS Appl. Mater. Interfaces 8, 23920-23931 (2016)

52. Zhang, J. T., Liu, P. P., Ma, H. Y. \& Ding, Y. Nanostructured porous gold for methanol electro-oxidation. J. Phys. Chem. C 111, 10382-10388 (2007).

53. Pedireddy, S. et al. Nanoporous gold bowls: a kinetic approach to control open shell structures and size-tunable lattice strain for electrocatalytic applications. Small 12, 4531-4540 (2016)

54. Yan, S. H. et al. Synthesis of $\mathrm{Au} / \mathrm{C}$ catalyst with high electrooxidation activity. Electrochim. Acta 94, 159-164 (2013).

55. Zhu, C. Z., Du, D., Eychmuller, A. \& Lin, Y. H. Engineering ordered and nonordered porous noble metal nanostructures: synthesis, assembly, and their applications in electrochemistry. Chem. Rev. 115, 8896-8943 (2015).

\section{Acknowledgements}

This work was supported by National Natural Science Foundation of China (Nos. 21675122 and 10434017), and the Key Research Program in Shaanxi (2017NY114). The authors thank Prof. D.Y. Zhao and C.L. Jia for helpful discussion.

\section{Author contributions}

L.L.Z. synthesized the materials and carried out the characterizations of the structures J.L., L.L., C.S.M. and S.D.C. completed the TEM, EDX Mapping and HR-TEM characterizations. H.J.Y. conducted the catalytic performance measurements. J.X.F., Z.Y.L. and Q.H.X. designed, supervised the project and wrote the manuscript. All authors discussed the results and commented on the manuscript.

\section{Additional information}

Supplementary Information accompanies this paper at https://doi.org/10.1038/s41467018-02930-9.

Competing interests: The authors declare no competing financial interests.

Reprints and permission information is available online at http://npg.nature.com/ reprintsandpermissions/

Publisher's note: Springer Nature remains neutral with regard to jurisdictional claims in published maps and institutional affiliations.

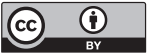

Open Access This article is licensed under a Creative Commons Attribution 4.0 International License, which permits use, sharing, adaptation, distribution and reproduction in any medium or format, as long as you give appropriate credit to the original author(s) and the source, provide a link to the Creative Commons license, and indicate if changes were made. The images or other third party material in this article are included in the article's Creative Commons license, unless indicated otherwise in a credit line to the material. If material is not included in the article's Creative Commons license and your intended use is not permitted by statutory regulation or exceeds the permitted use, you will need to obtain permission directly from the copyright holder. To view a copy of this license, visit http://creativecommons.org/ licenses/by/4.0/.

(C) The Author(s) 2018 